\title{
The X-ray emission mechanism in the protostellar jet HH 154
}

\author{
R. Bonito ${ }^{1}$, S. Orlando ${ }^{2}$, G. Peres ${ }^{1}$, F. Favata ${ }^{3}$, and R. Rosner ${ }^{4,5}$ \\ ${ }^{1}$ Dip. Scienze Fisiche ed Astronomiche, Sez. Astronomia, Università di Palermo, P.zza del Parlamento 1, 90134 Palermo, Italy \\ e-mail: sbonito@oapa.astropa.unipa.it \\ 2 INAF - Osservatorio Astronomico di Palermo, P.zza del Parlamento 1, 90134 Palermo, Italy \\ 3 Astrophys. Div. - Res. and Science Support Dept. of ESA, ESTEC, Postbus 299, 2200 AG Noordwijk, The Netherlands \\ ${ }^{4}$ Dept. of Astronomy and Astrophysics, Univ. of Chicago, Chicago, IL 60637, USA \\ 5 Center for Astrophysical Thermonuclear Flashes, University of Chicago, 5640 S. Ellis Avenue, Chicago, IL 60637, USA
}

Received 7 May 2004 / Accepted 24 June 2004

\begin{abstract}
We study the mechanism causing the X-ray emission recently detected in protostellar jets, by performing a detailed modeling of the interaction between a supersonic jet originating from a young stellar object and the ambient medium, for various values of density contrast, $v$, between the ambient density and the jet, and of Mach number, $M$; radiative losses and thermal conduction have been taken into account. Here we report a representative case which reproduces, without any ad hoc assumption, the characteristics of the X-ray emission recently observed in the protostellar jet HH 154. We find that the X-ray emission originates from a localized blob, consistent with observations, which moves with velocity $v \sim 500 \mathrm{~km} \mathrm{~s}^{-1}$; we therefore predict the X-ray source to have a detectable proper motion.
\end{abstract}

Key words. ISM: Herbig-Haro objects - ISM: individual objects: HH 154 - ISM: jets and outflows - X-rays: ISM

\section{Introduction}

Herbig-Haro $(\mathrm{HH})$ objects are mainly shocks produced at the interaction front between a protostellar supersonic jet and the ambient medium. Detailed studies of these objects in the radio, infrared and optical bands have been carried in the last 50 years, i.e. since HH objects were discovered (Herbig 1950; Haro 1952; see also Reipurth \& Bally 2001).

Recently the XMM-Newton and Chandra satellites have found evidence of X-ray emission from $\mathrm{HH}$ objects: $\mathrm{HH} 2$ in Orion (Pravdo et al. 2001), HH 154 in Taurus (Favata et al. 2002; Bally et al. 2003), HH 80/81 in the Sagittarius cloud L291 (Pravdo et al. 2004), and a protostellar jet in the OMC-3 molecular cloud in Orion (Tsujimoto et al. 2004).

$\mathrm{HH} 154$ is one of the nearest and best studied Herbig-Haro objects, and it is composed of several knots aligned along an optically visible jet leading away from the IRS 5 protostar. IRS 5 is a deeply embedded double source, obscured by about 150 mag of visual extinction and located in the L1551 cloud in Taurus, one of the nearest sites of ongoing star formation, at a distance of about $140 \mathrm{pc}$.

Favata et al. (2002) and Bally et al. (2003) discovered X-ray emission originating from $\mathrm{HH} 154$, unambiguously excluding that the X-ray source is associated with the L1551 IRS 5 protostar both because of the position of the X-ray source, displaced by $0.5-1$ arcsec from IRS 5 along the jet axis, and because the absorbing column density found from the X-ray spectrum (corresponding to a visual extinction $A_{\mathrm{V}} \approx 7 \mathrm{mag}$ ) is by far too small to be associated with IRS 5 ( $A_{\mathrm{V}} \approx 150 \mathrm{mag}$ ).
Several models have been proposed to explain the X-ray emission from protostellar jets, but the emission mechanism is still unclear. Bally et al. (2003), in particular, suggested various models, not based on simulations, for the X-ray emission from HH 154: these include X-ray emission from IRS 5 reflected by a dense medium, X-ray emission produced when the stellar wind shocks against the wind from the companion star, or produced in shocks in the jet. Raga et al. (2002) derived an analytic model and performed numerical simulations, predicting X-ray emission originating from the jet.

Prompted by this new X-ray-observed phenomenon, we studied a set of different physical configurations for the interaction between a supersonic jet and the ambient medium. We aimed to infer the configuration(s) which can give rise to $\mathrm{X}$-ray emission, to determine the range of parameters consistent with observations and to get insight on the jet physical conditions. To this end, we used the FLASH code (Fryxell et al. 2000) with customized numerical modules that treat optically thin radiative losses and thermal conduction. FLASH uses the PARAMESH library to handle adaptive mesh refinement (MacNeice et al. 2000) and the Message-Passing Interface library to achieve parallelization.

Here we present a first set of results concerning a jet less dense than the ambient medium, with density contrast $v=$ $n_{\mathrm{a}} / n_{\mathrm{j}}=10$ (where $n_{\mathrm{a}}$ is the ambient density and $n_{\mathrm{j}}$ is the density of the jet) which produces a good agreement with the observed X-ray emission from HH 154 (Favata et al. 2002). In a forthcoming paper we will study the effects of varying the 
parameters, such as the ambient-to-jet density ratio, $v$, and the Mach number, $M$, on the jet dynamics.

\section{The model}

We model a jet with density $n_{\mathrm{j}}=500 \mathrm{~cm}^{-3}$ and which travels through an ambient medium 10 times denser according to Fridlund \& Liseau (1998); the jet has high Mach number, $M=300$, corresponding to an initial velocity $v_{\mathrm{j}} \sim 1.4 \times$ $10^{3} \mathrm{~km} \mathrm{~s}^{-1}$, and temperature $T_{\mathrm{j}}=10^{4} \mathrm{~K}$. We assume that at the beginning the jet is in pressure equilibrium with its environment. The model takes into account optically thin radiative losses (Raymond \& Smith 1977 and subsequent upgrades; Mewe et al. 1985) and thermal conduction (Spitzer 1962) with saturation effects (Cowie \& McKee 1977); we assume that the role of magnetic field is irrelevant. The evolution of the protostellar jet is described by the fluid equations of mass, momentum and energy conservation.

We adopt a 2D cylindrical $(r, z)$ coordinate system with the jet axis coincident with the $z$-axis. The computational grid extends over $\approx 300 \mathrm{AU}$ in the $r$ direction and over $\approx 6500 \mathrm{AU}$ in the $z$ direction. The initial jet radius is $r_{\mathrm{j}} \approx 30 \mathrm{AU}$ and its initial length is $300 \mathrm{AU}$. Reflection boundary conditions are imposed along the jet axis, inflow boundary conditions at $z=0$ and $r \leq r_{\mathrm{j}}$ and outflow boundary conditions elsewhere. The maximum spatial resolution achieved is $\approx 1.3 \mathrm{AU}$ according to the PARAMESH methodology and using 4 refinement levels, corresponding to covering the jet radius with 25 points at the maximum resolution.

From the model, we derive the emission measure, EM, and temperature, $T$, for each fluid element; thus we obtain 3D maps of $E M$ and $T$, considering the axial symmetry. Following the approach of Orlando et al. (2000), we derive a distribution of emission measure vs. temperature, $\operatorname{EM}(T)$, over the temperature range $10^{3}-10^{8} \mathrm{~K}$ : the temperature range is first divided into 74 logarithmically spaced bins; then the emission measure of all the fluid elements corresponding to the same temperature bin is summed to yield the EM in that bin. We synthesize the focal plane spectrum as predicted to be observed with the Chandra/ACIS-I or XMM-Newton/EPIC-pn X-ray imaging spectrometers, using the Analysis System for Astrophysical Plasmas (ASAP; Maggio et al. 1994) suite of tools, as follows: from the $E M(T)$ distribution we synthesize the spectrum using the MEKAL spectral code (Mewe et al. 1985) taking into account the interstellar medium absorption column density, $N_{\mathrm{H}}$ (Morrison \& McCammon 1983); the absorbed parent spectrum is then folded through the spectral instrumental response, deriving the focal plane spectrum. Finally, we analyze the focal plane spectrum with XSPEC V11.2 and compare our findings with published experimental results (Favata et al. 2002).

\section{Results}

The analysis of ACIS-I data is interesting because of its high spatial resolution, which allows to localize the X-ray source in the jet with high precision (see Bally et al. 2003). Figure 1 shows the evolution of the temperature and of the X-ray emission integrated along the line-of-sight with a bin-size $~ 10 \mathrm{AU}$
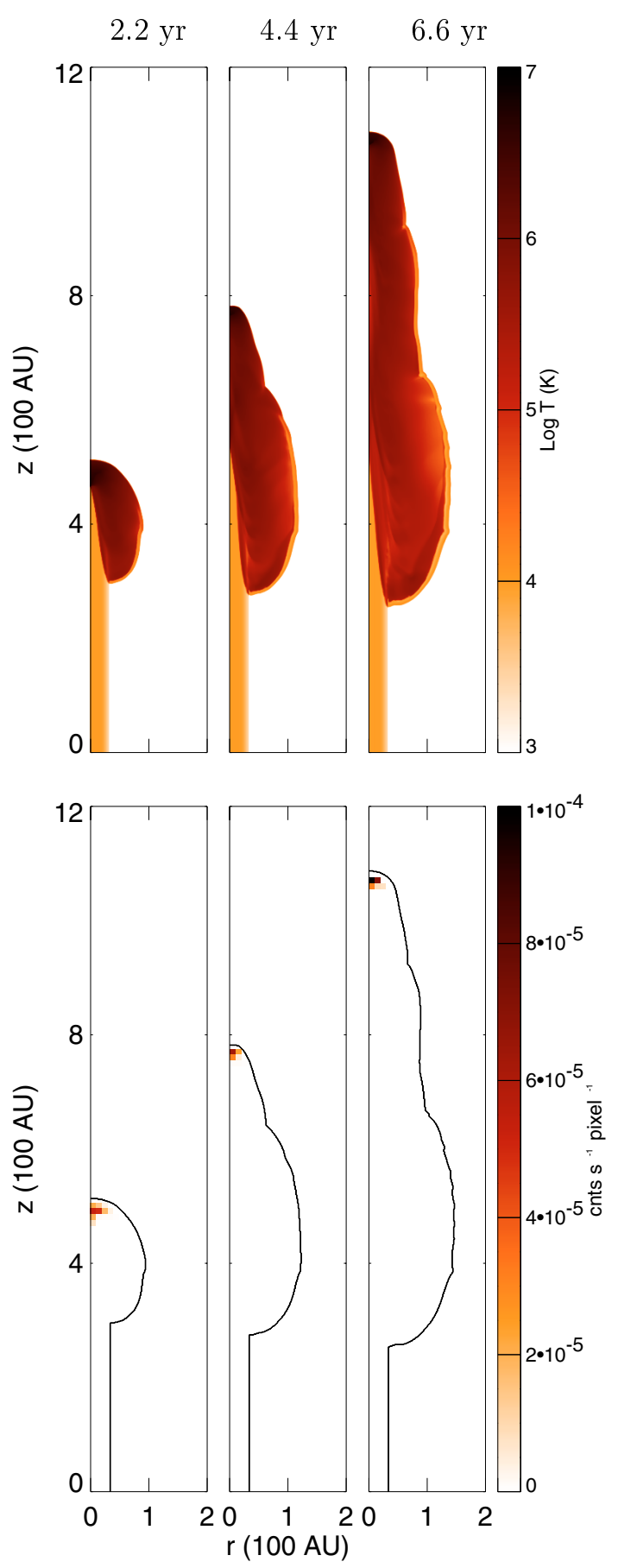

Fig. 1. Top panels: 2D cuts through the $r z$ plane of the jet temperature at three different evolutionary stages. Bottom panels: the corresponding X-ray emission integrated along the line-of-sight and on macropixels with $\sim 10$ AU of size (6 times better that the ACIS-I spatial resolution), as predicted to be observed with ACIS-I. The contour plot marks the region occupied by the jet and by the cocoon. The X-ray source is localized in a blob behind the bow shock, moving with measurable proper motion; its linear size is $\sim 30 \mathrm{AU}$.

(about 6 times better than Chandra spatial resolution), as predicted to be observed with ACIS-I, assuming that the jet propagates perpendicularly to the line-of-sight. Note the presence of a high temperature (up to $\sim 7 \times 10^{5} \mathrm{~K}$ ) cocoon (top panels) enveloping the jet; the temperature is almost uniform in 


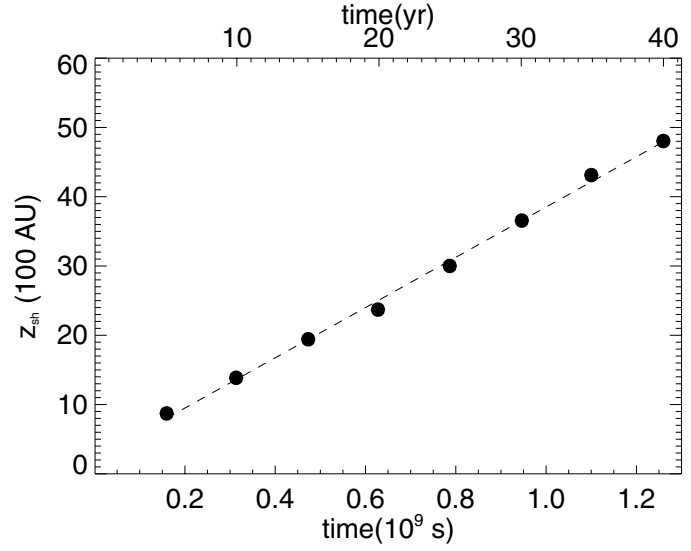

Fig. 2. Shock front position vs. time. The least-square fit (dashed line) gives an average velocity of the shock front of $v_{\mathrm{sh}} \sim 500 \mathrm{~km} \mathrm{~s}^{-1}$.

Table 1. Best-fit parameters to the EPIC-pn simulated X-ray spectra shown in Fig. 3 and obtained in the low and high statistics cases, respectively.

\begin{tabular}{llllll}
\hline \hline cnts & $\begin{array}{l}n_{\mathrm{H}} \pm \Delta n_{\mathrm{H}} \\
\left(10^{22} \mathrm{~cm}^{-3}\right)\end{array}$ & $\begin{array}{l}T \pm \Delta T \\
\left(10^{6} \mathrm{~K}\right)\end{array}$ & $\begin{array}{l}E M \pm \Delta E M \\
\left(10^{52} \mathrm{~cm}^{-3}\right)\end{array}$ & $\chi^{2}$ & Prob. $^{a}$ \\
\hline 83 & $1.50 \pm 0.36$ & $3.7 \pm 2.2$ & $1.4 \pm 6.0$ & 0.56 & 0.73 \\
$10^{4}$ & $1.37 \pm 0.02$ & $3.7 \pm 0.1$ & $1.3 \pm 0.3$ & 0.68 & 1.00 \\
\hline
\end{tabular}

${ }^{a}$ Null hypothesis probability.

the cocoon because of the thermal conduction. However the cocoon temperature decreases with time and gradually a cooler and denser shell forms. The X-ray emission (bottom panels) originates from a localized inner blob corresponding to the region behind the shock front, with a radius comparable to $r_{\mathrm{j}}$, i.e. $\sim 30 \mathrm{AU}$. From the values obtained for the average blob temperature, $T \sim 3.4 \times 10^{6} \mathrm{~K}$, and density, $n \sim 10^{4} \mathrm{~cm}^{-3}$, the cutoff length scale for thermal instability (Field 1965) is $\sim 10^{2}$ AU. Since this scale length exceeds the X-ray blob dimension, we can conclude that thermal conduction stabilizes the blob against radiation-driven thermal instability.

Figure 1 clearly shows that the $\mathrm{X}$-ray source moves in time along the jet. The shock front position, $z_{\mathrm{sh}}$, vs. time, $t$, is plotted in Fig. 2 together with the least-square fit to the linear model $z_{\mathrm{sh}}=A+v_{\mathrm{sh}} t$ : the shock front propagates with an average (and almost constant) velocity $v_{\mathrm{sh}} \sim 500 \mathrm{~km} \mathrm{~s}^{-1}$.

We synthesized the spectra as predicted to be observed with the EPIC-pn, in order to compare our analysis with the results obtained by Favata et al. (2002). We integrated the emission of the whole spatial domain (considering the 3D structure of $E M$ and $T)$, and we assumed two different statistics, low $\left(\sim 10^{2}\right.$ total counts, i.e. comparable to observations $)$ and high $\left(\sim 10^{4}\right.$ total counts). Assuming that the source is at a distance of $150 \mathrm{pc}$, we obtained exposure times ranging between 70 and $100 \mathrm{ks}$ in the low statistics case in the [0.3-10] keV band, with an absorption column density, $N_{\mathrm{H}}=1.4 \times 10^{22} \mathrm{~cm}^{-2}$, as in Favata et al. (2002). The energy channels are grouped together so as to have at least 10 photon counts per channel in the low statistics case and 20 photon counts per channel in the other case. The spectra, then, have been fitted with an isothermal MEKAL spectrum in
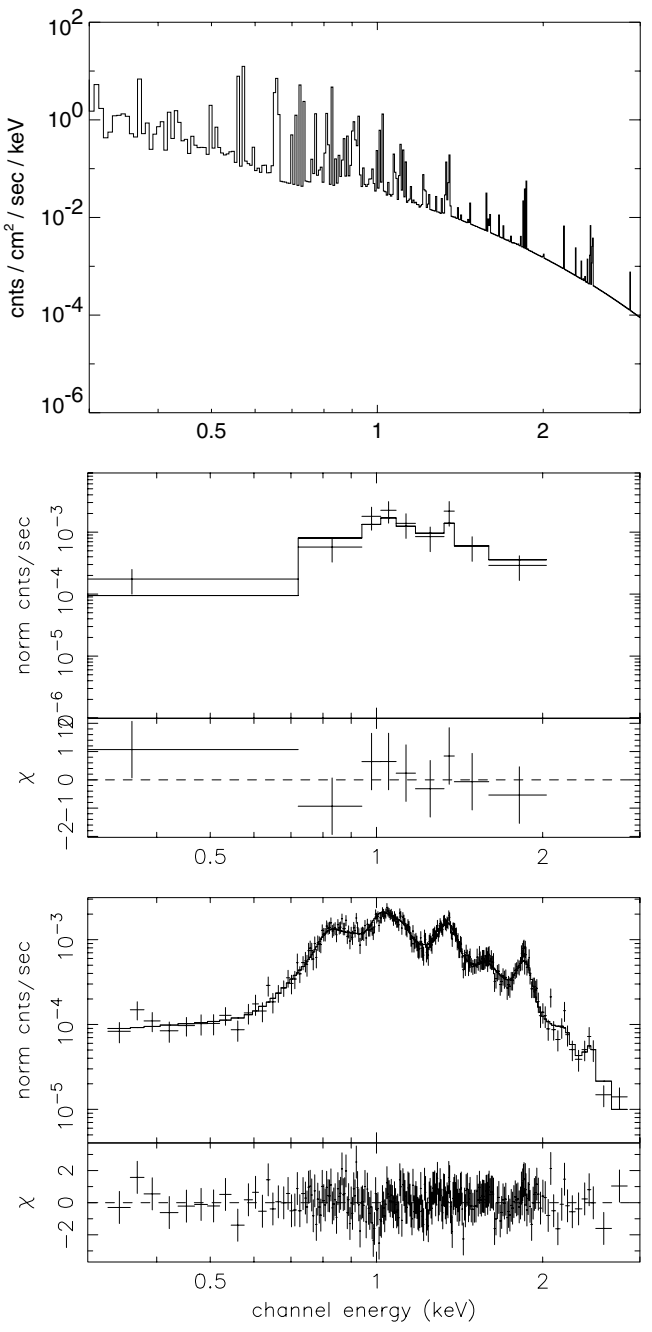

Fig. 3. Spectra in the $[0.3,3.0] \mathrm{keV}$ band synthesized with the MEKAL code from the original $E M(T)$ distributions of the whole spatial domain, 25 years since the beginning of the jet/ambient medium interaction. The top panel shows the unabsorbed parent spectrum; the middle and the bottom panels show the EPIC-pn spectra derived for two different statistics: $10^{2}$ and $10^{4}$ total counts respectively. For the EPIC-pn spectra each panel shows the simulated data (crosses) together with the best-fit isothermal spectrum (solid histograms) and the contribution of each bin to the total $\chi$. The corresponding best-fit parameters are in Table 1.

the [0.3-10] keV band, using the package XSPEC V11.2. As an example, in Fig. 3 we present the synthesized spectra, with the best-fit thermal (MEKAL) spectra superimposed, 25 years since the beginning of the jet/ambient medium interaction. The top panel shows the unabsorbed parent spectrum derived from the original $E M(T)$ distribution of the whole spatial domain; the middle and bottom panels show the EPIC-pn spectra derived in the low and high statistics cases, respectively. We found that all the spectra are well described as the emission from an optically thin plasma at a single temperature, even in the high statistics case; furthermore, the fitting parameters obtained in the low and high statistics cases are compatible among them (see examples in Table 1) and consistent with the observed values. From the best-fit isothermal component derived in the high statistic case, we obtain the X-ray luminosity, $L_{\mathrm{X}}$, in the EPIC-pn band; 


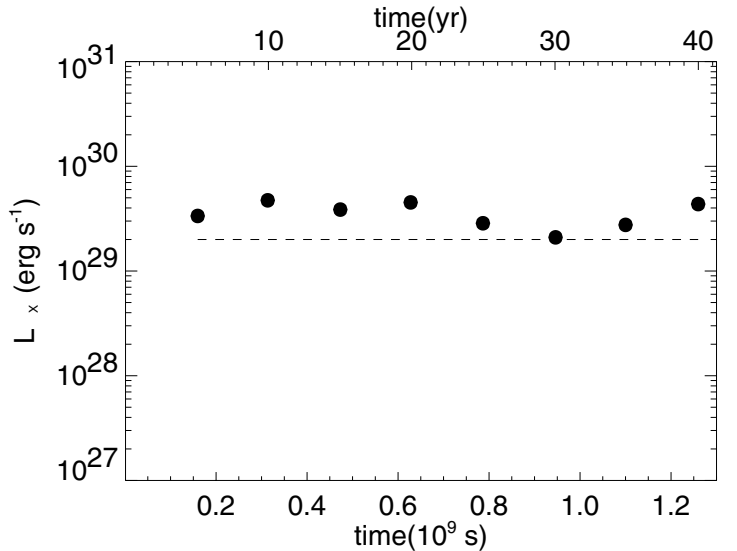

Fig. 4. Evolution of the X-ray luminosity vs. time. The value of the $\mathrm{X}$-ray luminosity derived from Raga et al. (2002) is superimposed (dashed line).

its evolution is shown in Fig. 4. We found that $L_{X}$ varies moderately, ranging between 2 and $5 \times 10^{29} \mathrm{erg} / \mathrm{s}$ during the simulated 40 years time span. The $L_{\mathrm{X}}$ values is consistent with that obtained in Favata et al. (2002) $\left(L_{\mathrm{X}} \approx 3 \times 10^{29} \mathrm{erg} / \mathrm{s}\right)$ and confirm the prediction of the simple analytic model proposed by Raga et al. (2002) $\left(L_{\mathrm{X}} \sim 2 \times 10^{29} \mathrm{erg} / \mathrm{s}\right.$, considering the radius of the blob, $r_{\mathrm{b}} \sim 30 \mathrm{AU}$, the ambient density, $n_{\mathrm{a}} \sim 5000 \mathrm{~cm}^{-3}$, and the shock front velocity, $v_{\mathrm{sh}} \sim 500 \mathrm{~km} \mathrm{~s}^{-1}$ ), in which the authors assume that the X-ray emitting region corresponds to the head of the bow shock, where the gas has a temperature and density of the order of the on-axis post-shock values. Our simulations confirm that assumption, demonstrating that the X-ray emission is effectively localized in a blob just behind the shock front. A crucial feature to achieve such good agreement with Raga et al. (2002) simpler model is the strong absorption of the interstellar medium which suppresses the soft X-ray emission component originating from the cocoon with temperature at slightly less than $10^{6} \mathrm{~K}$ (see Fig. 1). The absorption is also essential to fit the HH 154 observations.

\section{Discussion and conclusions}

In this letter we have shown our numerical simulations of the interaction between a protostellar jet and the ambient medium from which we derived many interesting features of the X-ray emission due to the formation of shocks at the interaction front between the jet and the ambient medium. Our model explains the X-ray emission observed in protostellar jets as emission from mechanical heating due to shocks produced by the interaction between the jet and the ambient medium.

Our simulations reproduce in a natural way, without any ad hoc assumption, the X-ray emission observed in HH 154 (Favata et al. 2002). In addiction, our model predicts that the X-ray emission originates in a localized blob with size $<100 \mathrm{AU}$, and that such a blob moves with velocity around $500 \mathrm{~km} \mathrm{~s}^{-1}$ (see Fig. 1). Our model, therefore, unambiguously predicts a measurable proper motion of the X-ray source which can be tested in future observations. This result is supported by previous optical studies which have analyzed the proper motions of knots along $\mathrm{HH} 154$, deriving spatial velocities of the order of $300 \mathrm{~km} \mathrm{~s}^{-1}$ (Sarcander et al. 1985; Neckel \& Staude 1987), and which have found high ionization clumps of material traveling at velocity of the order of $400-500 \mathrm{~km} \mathrm{~s}^{-1}$ near the base of the jet where the Chandra observation places the $\mathrm{X}$-ray source (Fridlund et al., in preparation).

Acknowledgements. We would like to thank M. Fridlund for his suggestions and helpful discussions and the referee, Dr. A. C. Raga, for helpful comments. The software used in this work was in part developed by the DOE-supported ASCI/Alliances Center for Astrophysical Thermonuclear Flashes at the University of Chicago, using modules for thermal conduction and optically thin radiation constructed at the Osservatorio Astronomico di Palermo. The calculations were performed on the Compaq cluster at the SCAN (Sistema di Calcolo per l'Astrofisica Numerica) facility of the Osservatorio Astronomico di Palermo and on the IBM/sp4 machine at CINECA (Bologna, Italy).

\section{References}

Bally, J., Feigelson, E., \& Reipurth, B. 2003, ApJ, 584, 843

Cowie, L. L., \& McKee, C. F. 1977, ApJ, 211, 135

Favata, F., Fridlund, C. V. M., Micela, G., Sciortino, S., \& Kaas, A. A. 2002, A\&A, 386, 204

Field, G. B. 1965, ApJ, 142, 531

Fridlund, C. V. M., \& Liseau, R. 1998, ApJ, 499, L75

Fryxell, B., Olson, K., Ricker, P., et al. 2000, ApJS, 131, 273

Haro, G. 1952, ApJ, 115, 572

Herbig, G. H. 1950, ApJ, 111, 11

MacNeice, P., Olson, K. M., Mobarry, C., de Fainchtein, R., \& Packer, C. 2000, Comp. Phys. Comm., 126, 330

Maggio, A., Reale, F., Peres, G., \& Ciaravella, A. 1994, Comp. Phys. Comm., 81, 105

Mewe, R., Gronenschild, E. H. B. M., \& van den Oord, G. H. J. 1985, A\&AS, 62, 197

Morrison, R., \& McCammon, D. 1983, ApJ, 270, 119

Neckel, T., \& Staude, H. J. 1987, ApJ, 322, L27

Orlando, S., Peres, G., \& Reale, F. 2000, ApJ, 528, 524

Pravdo, S. H., Feigelson, E. D., Garmire, G., et al. 2001, Nature, 413, 708

Pravdo, S. H., Tsuboi, Y., \& Maeda, Y. 2004, ApJ, 605, 259

Raga, A. C., Noriega-Crespo, A., \& Velázquez, P. F. 2002, ApJ, 576, L149

Raymond, J. C., \& Smith, B. W. 1977, ApJS, 35, 419

Reipurth, B., \& Bally, J. 2001, ARA\&A, 39, 403

Sarcander, M., Neckel, T., \& Elsaesser, H. 1985, ApJ, 288, L51

Spitzer, L. 1962, Physics of Fully Ionized Gases (New York: Interscience)

Tsujimoto, M., Koyama, K., Kobayashi, N., et al. 2004, PASJ, 56, 341 